\title{
On the Assessment of Tree-Based and Chance-Constrained Predictive Control Approaches applied to Drinking Water Networks ${ }^{\star}$
}

\author{
Juan M. Grosso* José M. Maestre** \\ Carlos Ocampo-Martinez * Vicenç Puig * \\ * Automatic Control Department, Universitat Politècnica de Catalunya \\ (UPC), Institut de Robòtica i Informàtica Industrial (CSIC-UPC), \\ Llorens i Artigas, 4-6, 08028 Barcelona, Spain \\ \{jgrosso, cocampo, vpuig\}@iri.upc.edu. \\ ** Departamento de Ingeniería de Sistemas y Automática, Universidad \\ de Sevilla, Escuela Superior de Ingenieros, Camino de los \\ Descubrimientos s/n, 41092 Sevilla, Spain \\ pepemaestre@us.es
}

\begin{abstract}
Water systems are a challenging problem because of their size and exposure to uncertain influences such as the unknown demands or the meteorological phenomena. In this paper, two different stochastic programming approaches are assessed when controlling a drinking water network: chance-constrained model predictive control (CC-MPC) and tree-based model predictive control (TB-MPC). Under the former approach, the disturbances are modelled as stochastic variables with non-stationary uncertainty description, unbounded support and quasiconcave probabilistic distribution. A deterministic equivalent of the related stochastic problem is formulated using Boole's inequality and a uniform allocation of risk. In the later approach, water demand is modelled as a disturbance rooted tree where branches are formed by the most probable evolutions of the demand. In both approaches, a model predictive controller is used to optimise the expectation of the operational cost of the disturbed system.
\end{abstract}

Keywords: control of water systems, model predictive control, stochastic programming.

\section{INTRODUCTION}

The management and control of water systems is a challenging problem due to different sources of uncertainty. The availability of historical data allows to accurately predict the behaviour of the system disturbances over large horizons but still a meaningful degree of uncertainty is present. Besides uncertainty, water systems often exhibit other complicating features such as multi-variable interactions, transport delays, hard constraints on the system variables, or multiple conflicting control goals. Due to all these problems, model predictive control (MPC) has been proposed as a suitable technique to control water systems, see a further discussion in OcampoMartinez et al. [2013]. By using MPC, it is possible to explicitly incorporate the aforementioned features into an optimisation problem in a systematic manner. To this end, a mathematical model of the system is used to predict its future behaviour, which is optimised in a receding horizon fashion according to a given performance index over a certain prediction horizon [Maciejowski, 2002].

\footnotetext{
* This research has been supported by the EU Project EFFINET (FP7-ICT-2011-8-31855), the EU Network of Excellence Highly Complex and Networked Control Systems (HYCON2) under grant agreement no. 257462, and the DGR of Generalitat de Catalunya (SAC group Ref. 2009/SGR/1491).
}

This paper focuses on the way that uncertainty can be faced from an MPC framework in the control of drinking water networks (DWN). The simplest way to do this is by ignoring the explicit influence of disturbances or using their expected value. Unfortunately, this approach may lead to poor control performance or frequent constraints violation. As discussed in Cannon et al. [2007], alternative approaches of MPC for stochastic systems are based on min-max MPC, tube-based MPC, and stochastic MPC. The first two consider disturbances to be unmeasured but bounded in a predefined set, which is more conservative and reduces the control performance due to the worst-case nature of the schemes. On the other hand, the stochastic MPC considers a more realistic description of uncertainty, which leads to less conservative control approaches at the expense of a more complex modelling of the disturbances. The stochastic approach has a mature theory in the field of optimisation [Calafiore and Dabbene, 2006], but a renewed attention has been given to the stochastic programming techniques as powerful tools for control design, see, e.g., Calafiore et al. [2011] and references therein.

From the wide range of stochastic MPC methods, this paper focuses on tree-based MPC (TB-MPC) and chanceconstrained MPC (CC-MPC). Regarding TB-MPC, see, e.g., Raso et al. [2009] and Lucia et al. [2013], uncertainty 
is addressed by considering simultaneously a set of possible disturbance scenarios modelled as a rooted tree, which branches along the prediction horizon. On the other hand, CC-MPC [Schwarm and Nikolaou, 1999] is a stochastic control strategy that describes robustness in terms of probabilistic (chance) constraints, which require that the probability of violation of any operational requirement or physical constraint is below a prescribed value, representing the notion of reliability or risk of the system. By setting this value properly, the operator/user can trade conservatism against performance. Relevant works that address the CC-MPC approach in water systems can be found in Geletu et al. [2013], Ouarda and Labadie [2001] and references therein.

The main contribution of this paper consists in the design and assessment of TB-MPC and CC-MPC controllers applied for the operational management of a drinking water network (DWN), discussing their advantages and weakness in the sense of applicability and performance. The particular case study is related to the DWN of Barcelona (Spain).

The reminder of the paper is organised as follows. Section 2 states the control problem, describing its formulation within both TB-MPC and CC-MPC frameworks addressed in this paper. Section 3 describes the case study consisting in a small DWN, where the proposed approaches are evaluated. Section 4 highlights the concluding remarks and some future research directions.

\section{STOCHASTIC MPC FORMULATIONS}

\subsection{Problem Statement}

MPC uses a mathematical model to calculate the optimal control actions according to a given cost function [Maciejowski, 2002]. In this paper, it is assumed that the system behaviour can be described at each time instant $k \in \mathbb{Z}$ by the following discrete-time difference equation:

$$
x_{k+1}=A x_{k}+B u_{k}+E w_{k},
$$

where $x_{k} \in \mathbb{R}^{n_{x}}$ is the state of the system, $u \in \mathbb{R}^{n_{u}}$ is the vector of manipulated variables, and $w \in \mathbb{R}^{n_{w}}$ is a vector of measurable disturbances. Moreover, $A, B$, and $E$ are time-invariant matrices of proper dimensions. It is also considered that the system is subject to hard state and input constraints, which can be posed as

$$
\begin{aligned}
& x \in \mathcal{X} \triangleq\left\{x_{k} \in \mathbb{R}^{n_{x}} \mid G x_{k} \leq g, \forall k\right\}, \\
& u \in \mathcal{U} \triangleq\left\{u_{k} \in \mathbb{R}^{n_{u}} \mid F u_{k} \leq f, \forall k\right\},
\end{aligned}
$$

where $\mathcal{X}$ and $\mathcal{U}$ are closed polyhedra defined by a system of linear inequalities. In this sense, $G \in \mathbb{R}^{c_{x} \times n_{x}}, g \in \mathbb{R}^{c_{x}}$, $F \in \mathbb{R}^{c_{u} \times n_{u}}, f \in \mathbb{R}^{c_{u}}$, being $c_{x}$ and $c_{u}$ the number of state and input constraints, respectively.

The control goal is to minimise a convex (possible multi-objective) cost function $\ell(x, u): \mathcal{X} \times \mathcal{U} \rightarrow \mathbb{R}$, which might bear any functional relationship to the operating cost of the system. From the model in (1), let $w_{[k: k+N-1]} \triangleq\left(w_{k}, w_{k+1 \mid k}, \ldots, w_{k+N-1 \mid k}\right)$ be the sequence of disturbances over a fixed time prediction horizon $N \in \mathbb{Z}_{+}$. The first element of the sequence is measured, while the rest of the elements, i.e., $w_{k+i \mid k}$, denote estimates of future disturbances computed by an exogenous system and available at each time instant $k$. Hence, the MPC controller design is based on the solution of the following finite horizon optimisation problem (FHOP):

$$
\min _{\bar{u}_{[0: N-1]}} \sum_{i=0}^{N-1} \ell\left(\bar{x}_{i}, \bar{u}_{i}\right)
$$

subject to:

$$
\begin{aligned}
& \bar{x}_{i+1}=A \bar{x}_{i}+B \bar{u}_{i}+E \bar{w}_{i}, \\
& \bar{x}_{i+1} \in \mathcal{X}, \\
& \bar{u}_{i} \in \mathcal{U}, \\
& \bar{x}_{0}=x_{k}, \quad \bar{w}_{0}=w_{k}, \\
& \bar{w}_{i}=w_{k+i \mid k} \quad \forall i \in \mathbb{Z}_{1}^{N-1},
\end{aligned}
$$

where vectors $\bar{x}, \bar{u}$, and $\bar{w}$ denote the predicted value of the states, inputs and measured disturbances at the prediction step $i$. Notation $\mathbb{Z}_{a}^{b}$ expresses the set of integer numbers from $a$ to $b$, both limits included, i.e., $\{a, a+1, \ldots, b\}$.

Assuming that (3) is feasible, i.e., there exists a non-empty solution given by the optimal sequence of control inputs $\bar{u}_{[0: N-1]}^{*} \triangleq\left(\bar{u}_{0}^{*}, \bar{u}_{1}^{*}, \ldots, \bar{u}_{N-1}^{*}\right)$, then the receding horizon philosophy commands to apply the control action

$$
u_{k}=\kappa_{\mathrm{mpc}}\left(x_{k}, w_{[k: k+N-1]}\right)=\bar{u}_{0}^{*},
$$

and disregards the rest of the sequence of the predicted manipulated variables. At the next time instant $k$, the FHOP (3) is solved again using the current measurements of states and disturbances and the most recent forecast of these latter over the next future horizon.

Given the stochastic nature of future disturbances, the prediction model (3b) involves exogenous additive uncertainty, hence, the compliance of constraints for a given control input cannot be ensured. This means that, even if the predictive controller finds a feasible solution to achieve the operational goals, there is a certain probability that real outputs may violate constraints. Therefore, the use of SMPC strategies may allow to establish a trade-off between robustness and performance.

\section{2 $C C-M P C$}

As pointed out before, the optimal solution to (3) does not always imply feasibility of the real system due to the stochastic nature of disturbances. Therefore, it is appropriate to relax the original constraints in (2a) with probabilistic statements in the form of chance constraints. In this way, state constraints are required to be satisfied with a predefined probability to manage the reliability of the system as follows:

$$
\mathbb{P}\left[x_{k} \in \mathcal{X}\right] \geq 1-\delta_{x}, \quad \forall k \in \mathbb{Z}_{+},
$$

where $\mathbb{P}$ denotes the probability operator and $\delta_{x} \in(0,1)$ is the risk acceptability level of constraint violation for the states. Let (5) be rewritten as

$$
\mathbb{P}\left[G_{(j)} x_{k} \leq g_{(j)}, \forall j \in \mathbb{Z}_{1}^{c_{x}}\right] \geq 1-\delta_{x}, \quad \forall k \in \mathbb{Z}_{+},
$$

where $G_{(j)}$ and $g_{(j)}$ denote the $j^{\text {th }}$ row of $G$ and $g$, respectively. Hence, this probabilistic constraint is in the form of the so called joint chance constraint, which requires that the $c_{x}$ element-wise inequalities have to be jointly fulfilled with the given probability at each time instant $i$.

In general, joint chance constraints lack from analytic expressions due to the involved multivariate probability distribution. Nevertheless, sampling-based methods, 
numeric integration, and convex analytic approximations exists, see e.g., Calafiore and Dabbene [2006] and references therein. In this paper, (6) is approximated following the results in Prékopa [1995] and Nemirovski and Shapiro [2006] by upper bounding the joint constraint and assuming a uniform distribution of the joint risk among a set of individual chance constraints that are later transformed into equivalent deterministic constraints under the following consideration:

Assumption 1. Each disturbance in $w$ follows a logconcave univariate distribution, which stochastic description is known.

Given the dynamic model in (1), the stochastic nature of the disturbances $w$ makes the states $x$ to be also stochastic variables. Then, it follows for all time instants that

$$
F_{G x}(g)=\mathbb{P}\left[\left\{G_{(1)} x \leq g_{(1)}, \ldots, G_{\left(c_{x}\right)} x \leq g_{\left(c_{x}\right)}\right\}\right] .
$$

Defining the events $C_{j} \triangleq\left\{G_{(j)} x \leq g_{(j)},\right\}, \forall j \in \mathbb{Z}_{1}^{c_{x}}$, and their complements $C_{j}^{c} \triangleq\left\{G_{(j)} x>g_{(j)},\right\}$, then it follows that

$$
F_{G x}(g)=1-\mathbb{P}\left[\left(C_{1}^{c} \cup \ldots \cup C_{c_{x}}^{c}\right)\right] \geq 1-\delta_{x} .
$$

Taking advantage of the union bound, the Boole's inequality allows to bound the probability of the second term in the left-hand side of (8), stating that the probability that at least one event happens is no longer than the sum of the individual probabilities [Prékopa, 1995]. Hence, it follows that

$$
\sum_{j=1}^{c_{x}} \mathbb{P}\left[C_{j}^{c}\right] \leq \delta_{x} \Leftrightarrow \sum_{j=1}^{c_{x}}\left(1-\mathbb{P}\left[C_{j}\right]\right) \leq \delta_{x} .
$$

At this point, a set of constraints arise from previous result as sufficient conditions to enforce the joint chance constraint (6), by allocating the joint risk $\delta_{x}$ in $c_{x}$ separate risks $\delta_{x, j}, j \in \mathbb{Z}_{1}^{c_{x}}$. These constraints are:

$$
\begin{aligned}
& \mathbb{P}\left[C_{j}\right] \geq 1-\delta_{x, j}, \quad \forall j \in \mathbb{Z}_{1}^{c_{x}}, \\
& \sum_{j=1}^{c_{x}} \delta_{x, j} \leq \delta_{x}, \\
& 0 \leq \delta_{x, j} \leq 1,
\end{aligned}
$$

where (10) forms the set of $c_{x}$ resultant individual chance constraints, which bounds the probability that each inequality of the receding horizon problem may fail; and (11) and (12) are conditions imposed to bound the new single risks in such a way that the joint risk bound is not violated. Any solution that satisfies the above constraints, is guaranteed to satisfy (6). Assigning, e.g., a fixed and equal value of risk to each individual constraint, i.e., $\delta_{x, j}=\delta_{x} / c_{x}$, satisfies (11) and (12) [Nemirovski and Shapiro, 2006].

After decomposing the joint constraints into a set of individual constraints, the deterministic equivalent of each separate constraint may be used given that the probabilistic statements are not suitable for algebraic solution. Such deterministic equivalents might be obtained following Charnes and Cooper [1963]. Assuming a known (or approximated) quasi-concave probabilistic distribution $F_{E w}(b)=\mathbb{P}[E w \leq b]$ for the effect of the stochastic disturbance in the dynamic model (1), then it follows that

$$
\begin{aligned}
& \mathbb{P}\left[G_{(j)} x_{k+1} \leq g_{(j)}\right] \geq 1-\delta_{x, j} \\
& \Leftrightarrow G_{(j)}\left(A x_{k}+B u_{k}\right) \leq g_{(j)}-z_{\delta_{x, j}},
\end{aligned}
$$

where $z_{\delta_{x, j}} \triangleq F_{G_{(j)} E w_{k}}^{-1}\left(1-\delta_{x} / c_{x}\right)$ is the quantile function of $G_{(j)} E w_{k}$. Given that the state $x$ is also a stochastic variable, the above deterministic equivalent should be considered in terms of the expectation of the variables. In this way, the original constrained set $\mathcal{X}$ is contracted with the effect of the $c_{x}$ deterministic equivalents and replaced in $(3 \mathrm{c})$ with the stochastic feasibility set $\mathcal{X}_{s}$ given by

$$
\begin{aligned}
\mathcal{X}_{s} \triangleq\{ & \bar{x} \in \mathbb{R}^{n_{x}} \mid \exists u \in \mathcal{U}, \\
& \left.G_{(j)}\left(A \bar{x}_{k}+B \bar{u}_{k}\right) \leq g_{(j)}-z_{\delta_{x, j}}\right\}, \forall j \in \mathbb{Z}_{1}^{c_{x}} .
\end{aligned}
$$

The reformulated predictive controller solves the following deterministic equivalent FHOP for the expectation $\mathbb{E}[\cdot]$ of the cost function in (3a):

$$
\min _{\bar{u}(0: N-1)} \sum_{i=0}^{N-1} \mathbb{E}\left[\ell\left(\bar{x}_{i}, \bar{u}_{i}\right)\right]
$$

subject to:

$$
\begin{aligned}
& (3 \mathrm{~b}),(3 \mathrm{~d}),(3 \mathrm{e}),(3 \mathrm{f}), \\
& \bar{x}_{i+1} \in \mathcal{X}_{s} .
\end{aligned}
$$

\section{$2.3 T B-M P C$}

The deterministic equivalent CC-MPC proposed before might be still conservative if the probabilistic distributions of the stochastic variables are not well characterized or do not lie in a log-concave form. Therefore, this section presents a TB-MPC strategy that relies in scenario-trees to approximate the original problem, dropping Assumption 1. The approach followed by TB-MPC is based on modelling the possible scenarios of the disturbances as a rooted tree. This means that all the scenarios start from the same expected disturbance value. From that point, the scenarios must remain equal until the point in which they diverge from each other, which is called a bifurcation point. For instance, consider two scenarios $a$ and $b$ defined by the sequences $w_{[0: N-1]}^{a}$ and $w_{[0: N-1]}^{b}$ and let $k_{\mathrm{bp}}$ be the moment in which they diverge, then $w_{k}^{a}=w_{k}^{b}$ for $k \leq k_{\mathrm{bp}}$ and $w_{k}^{a} \neq w_{k}^{b}$ for $k>k_{\mathrm{bp}}$.

Notice that before a bifurcation point, the evolution followed by the disturbance cannot be anticipated because different evolutions are possible. For this reason, the controller has to calculate control actions that are valid for all the scenarios in the branch. Once the bifurcation point has been reached, the uncertainty is solved and the controller can calculate specific control actions for the scenarios in each of the new branches. Hence, the outcome of TB-MPC is not a single sequence of control actions, but a tree with the same structure of that of the disturbances. As in standard MPC, only the first element of this tree is applied (the root) and the problem is repeated in a receding horizon fashion.

The easiest way to understand the optimisation problem that has to be solved in TB-MPC is to solve as many instances of Problem (3) as the number $N_{s}$ of scenarios considered. Due to the increasing uncertainty, it is necessary to include non-anticipativity constraints [Rockafellar and Wets, 1991] in the MPC formulation so that the calculated input sequence is always ready to face any possible future bifurcation in the tree. More specifically, if $u_{[0: N-1]}^{a}$ and $u_{[0: N-1]}^{b}$ are the input sequences that correspond respectively to the scenarios $a$ and $b$ calculated 


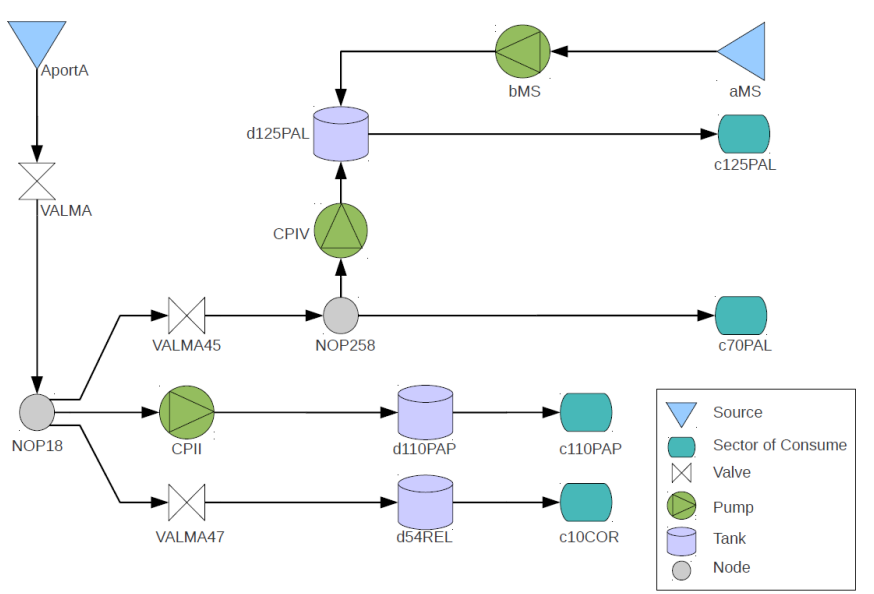

Figure 1. Topology of the Three-tanks DWN example

according to Problem (3), then the non-anticipativity constraint $u_{k}^{a}=u_{k}^{b}$ has to be satisfied whenever $w_{k}^{a}=w_{k}^{b}$ in order to guarantee that the set of inputs $u_{[0: N-1]}^{j}$ with $j \in \mathbb{Z}_{1}^{N_{s}}$ forms a tree with the same structure of that of the disturbances.

In this way, the TB-MPC controller has to solve the following FHOP, accounting for the $N_{s} \in \mathbb{Z}_{+}$scenarios, each with probability $p_{j} \in[0,1]$ and $\sum_{j=1}^{N_{s}} p_{j}=1$ :

$$
\min _{\bar{u}_{[0: N-1]}^{j}} \sum_{j=1}^{N_{s}}\left(p_{j} \sum_{i=0}^{N-1} \ell\left(\bar{x}_{i}^{j}, \bar{u}_{i}^{j}\right)\right)
$$

subject to:

$$
\begin{aligned}
& \bar{x}_{i+1}^{j}=A \bar{x}_{i}^{j}+B \bar{u}_{i}^{j}+E \bar{w}_{i}^{j}, \\
& \bar{x}_{i+1}^{j} \in \mathcal{X}, \\
& \bar{u}_{i}^{j} \in \mathcal{U}, \\
& \bar{x}_{0}^{j}=x_{k}, \quad \bar{w}_{0}^{j}=w_{k}, \\
& \bar{w}_{i}^{j}=w_{k+i \mid k}^{j}, \quad \forall i \in \mathbb{Z}_{1}^{N-1}, \\
& \bar{u}_{i}^{a}=\bar{u}_{i}^{b} \quad \text { if } \quad \bar{w}_{i}^{a}=\bar{w}_{i}^{b}, \quad \forall a, b \in \mathbb{Z}_{1}^{N_{s}} .
\end{aligned}
$$

Remark 1. The number of scenarios used to build the rooted tree should be determined regarding the computational capacity and the probability of risk that the manager is willing to accept.

\section{A DRINKING WATER NETWORK CASE STUDY}

\subsection{Case Study Description}

This section briefly describes a motivational example useful to assess SMPC approaches to solve multi-objective control problems attained to DWNs subject to stochastic disturbances and constraints. The case study consists of a small but representative example of a DWN that has to supply certain water demands by making optimal use of water sources and network components in order to minimise economic costs and guarantee service reliability. In general, the DWN operation is driven by the electricity prices and the exogenous and endogenous demands. The system under study is a portion extracted from the Barcelona DWN reported in Ocampo-Martinez et al. [2013]. In Fig. 1, a schematic representation of the DWN example is shown, which contains two water sources, three tanks, six manipulated actuators, four demand sectors and two intersection nodes.

\subsection{Control-oriented Model}

Consider a directed graph abstracted from Fig. 1. Setting the volume in storage tanks as the state variables $x \in \mathbb{R}^{n_{x}}$, the flow through the actuators as the manipulated inputs $u \in \mathbb{R}^{n_{u}}$, and the demanded flows as additive measured disturbances $d \in \mathbb{R}^{n_{d}}$, then the control-oriented model of the network may be abstracted from the connectivity analysis and described by the following set of discrete difference-algebraic equations for all time instant $k \in \mathbb{Z}_{+}$:

$$
\begin{aligned}
x_{k+1} & =A x_{k}+B u_{k}+B d_{k}, \\
0 & =E_{u} u_{k}+E_{d} d_{k},
\end{aligned}
$$

where (17a) and (17b) describe the mass balance equations for storage tanks and intersection nodes, respectively. Moreover, $A, B, B_{p}, E_{u}$ and $E_{d}$, are time-invariant matrices of dimensions dictated by the network topology. Assumption 2. The states in $x$ and the demands in $d$ are observable at time $k$, and the pair $(A, B)$ is controllable.

Assumption 3. The realisation of disturbances at the current time instant $k$ may be decomposed as

$$
d_{k}=\bar{d}_{k}+e_{k}
$$

where $\bar{d}_{k} \in \mathbb{R}^{n_{d}}$ is the vector of expected disturbances, and $e_{k} \in \mathbb{R}^{n_{d}}$ is the vector of forecasting errors with nonstationary uncertainty and a known (or approximated) quasi-concave probability distribution $\mathcal{D}$. Therefore, the stochastic nature of each $j^{\text {th }}$ row of $d_{k}$ is described by $d_{(j), k} \sim \mathcal{D}_{i}\left(\bar{d}_{(j), k}, \Sigma\left(e_{(j), k}\right)\right)$, where $\bar{d}_{(j), k}$ denotes its mean, and $\Sigma\left(e_{(j), k}\right)$ its variance.

The system is subject to hard state and input constraints given by the following convex and closed polytopic sets:

$$
\begin{aligned}
& \mathcal{X} \triangleq\left\{x_{k} \in \mathbb{R}^{n_{x}} \mid x_{\min } \leq x_{k} \leq x_{\max }\right\}, \quad \forall k, \\
& \mathcal{U} \triangleq\left\{u_{k} \in \mathbb{R}^{n_{u}} \mid u_{\min } \leq u_{k} \leq u_{\max }\right\}, \quad \forall k,
\end{aligned}
$$

where $x_{\min } \in \mathbb{R}^{n_{x}}$ and $x_{\max } \in \mathbb{R}^{n_{x}}$ denote the vectors of minimum and maximum volume capacities in tanks, respectively, given in $\mathrm{m}^{3}$; while $u_{\text {min }} \in \mathbb{R}^{n_{u}}$ and $u_{\max } \in \mathbb{R}^{n_{u}}$ denote the vectors of minimum and maximum flow capacities through the system actuators, respectively, given in $\mathrm{m}^{3} / \mathrm{s}$. Moreover, for safety and supply service reliability, the states are subject to the following management soft constraint:

$$
x_{k} \geq s_{k}-\xi_{k} \geq 0, \quad \forall k,
$$

where $s_{k} \in \mathbb{R}_{+}^{n_{x}}$ is a positive vector of base stocks (minimal volume in each tank to avoid stock-outs) and $\xi_{k} \in \mathbb{R}_{+}^{n_{x}}$ is a vector of positive slack variables to be minimised, which represent the amount of water volume in tanks that is allowed to go below the desired base stocks.

\subsection{Demand Modelling and Scenario Generation}

In DWNs, the uncertainty is generally introduced by the unpredictable behaviour of water consumers. Therefore, a proper demand modelling is required to achieve an acceptable water supply service level. For the case study of 
this paper, time series forecasting based on auto-regressive integrated moving average (ARIMA) models is used due to its ability to capture complex linear dynamics from historical data [Box et al., 1994]. Once these models are calibrated, they are used here to generate a large number of possible demand scenarios by Monte Carlo sampling for a given prediction horizon $N \in \mathbb{Z}_{+}$. For the CC-MPC approach the mean demand path is used, while for the TB-MPC approach a set of scenarios is selected. A large number of scenarios might improve the robustness of the TB-MPC approach but at the cost of additional computational burden and economic performance losses. Hence, a trade-off must be achieved between performance and computational burden. To this end, a representative subset of scenarios may be chosen using scenario reduction algorithms. In this paper, the backward reduction algorithm in Heitsch and Römisch [2009] is used to reduce a specified initial fan of $N_{s}$ equally probable scenarios into a rooted tree of $N_{r}$ scenarios.

\subsection{Control Problem Formulation}

The formulation of the SMPC problems for the above case study should address the design of a control law $u_{k}=\kappa\left(x_{k}, d_{[k: k+N-1]}\right)$ that minimises, in a receding horizon fashion, the following cost function:

$$
\ell_{k} \triangleq \lambda_{1} \ell^{e}\left(u_{k}, \alpha_{k}\right)+\lambda_{2} \ell^{s}\left(\xi_{k}\right)+\lambda_{3} \ell^{\Delta}\left(\Delta u_{k}\right),
$$

where $\ell^{e}\left(u_{k}, \alpha_{k}\right) \triangleq \alpha_{k}^{\top} W_{e} u_{k} \in \mathbb{R}_{>0}$ represents the economic cost of network operation that depends on a time-of-use pricing scheme given by $\alpha_{k} \triangleq\left(\alpha_{1}+\alpha_{2, k}\right) \in$ $\mathbb{R}^{n_{u}}$, which takes into account a fixed water production cost $\alpha_{1} \in \mathbb{R}^{n_{u}}$ and a time-varying water pumping cost $\alpha_{2, k} \in \mathbb{R}^{n_{u}}$ that changes according to the electric tariff; $\ell^{s}\left(\xi_{k}\right) \triangleq \xi_{k}^{\top} W_{s} \xi_{k} \in \mathbb{R}_{\geq 0}$ is a performance index that penalises the amount of volume $\xi$ going below from the $s$ threshold; and $\ell^{\Delta}\left(\Delta u_{k}\right) \triangleq \Delta u_{k}^{\top} W_{\Delta u} \Delta u_{k} \in \mathbb{R}_{\geq 0}$ represents the penalisation of control signal variations $\Delta u_{k} \triangleq u_{k}-u_{k-1}$, to extend actuators life and assure a smooth operation. Furthermore, $W_{e}, W_{s}, W_{\Delta u}$ are diagonal positive definite matrices that weight each decision variable in their corresponding cost function, and $\lambda_{1}, \lambda_{2}, \lambda_{3}$ are positive scalar weights that allow to prioritise the impact of each involved objective on the overall performance of the system. These weights are assumed to be fixed by the managers of the DWN. Regarding constraint (20), the minimal volume of water required in a tank is given by its net demand, hence, the minimal value of $x$ should be ideally given by $s_{k}=-B_{p} d_{k}$. For the TB-MPC approach, this minimal volume applies for each $j \in \mathbb{Z}_{1}^{N_{s}}$ prediction model, i.e., $\bar{x}_{i}^{j} \geq-B_{p} \bar{d}_{i}^{j}-\xi_{i}^{j}$ for all $i$ prediction steps. In this approach, robustness comes from the fact of considering $N_{s}$ scenarios simultaneously. In contrast, the deterministic equivalent CC-MPC approach automatically computes a minimal volume given by the net predicted demand plus a robustness factor that depends on the forecast variance and the risk acceptability level as shown in (14).

\subsection{Results}

Numeric results of applying the deterministic equivalent CC-MPC and TB-MPC discussed in Section 2 to the
Table 1. Assessment of the CC-MPC and TB-MPC applied to the DWN case study.

\begin{tabular}{|c|c|c|c|c|c|c|c|c|c|c|}
\hline \multicolumn{5}{|c|}{ CC-MPC } & \multicolumn{6}{|c|}{ TB-MPC } \\
\hline$\delta$ & $\Phi_{1}$ & $\Phi_{2}$ & $\Phi_{3}$ & $\Phi_{4}$ & $\Phi_{1}$ & $\Phi_{2}$ & $\Phi_{3}$ & $\Phi_{4}$ & $N_{r}$ & $N_{s}$ \\
\hline \multirow{3}{*}{0.3} & \multirow{3}{*}{58535.80} & \multirow{3}{*}{0} & \multirow{3}{*}{0} & \multirow{3}{*}{1.246} & 58397.14 & 0 & 0 & 0.940 & 5 & \multirow{3}{*}{19} \\
\hline & & & & & 58280.69 & 1 & 0.510 & 1.607 & 10 & \\
\hline & & & & & 58279.95 & 1 & 4.155 & 2.373 & 14 & \\
\hline \multirow{3}{*}{0.2} & \multirow{3}{*}{58541.19} & \multirow{3}{*}{0} & \multirow{3}{*}{0} & \multirow{3}{*}{1.208} & | 58482.14 & 3 & 0.183 & 1.178 & 7 & \multirow{3}{*}{29} \\
\hline & & & & & 58903.63 & 0 & 0 & 2.326 & 14 & \\
\hline & & & & & 58452.41 & 0 & 0 & 4.048 & 21 & \\
\hline \multirow{3}{*}{0.1} & \multirow{3}{*}{58558.29} & \multirow{3}{*}{0} & \multirow{3}{*}{0} & \multirow{3}{*}{1.252} & |58610.32 & 0 & 0 & 2.570 & 14 & \multirow{3}{*}{59} \\
\hline & & & & & 58630.20 & 0 & 0 & 6.655 & 29 & \\
\hline & & & & & 58656.56 & 1 & 0.178 & 13.466 & 44 & \\
\hline \multirow{3}{*}{0.01} & \multirow{3}{*}{58612.28} & \multirow{3}{*}{0} & \multirow{3}{*}{0} & \multirow{3}{*}{1.253} & - & - & - & - & 149 & \multirow{3}{*}{599} \\
\hline & & & & & - & - & - & - & 299 & \\
\hline & & & & & - & - & - & - & 449 & \\
\hline \multirow{3}{*}{0.001} & \multirow{3}{*}{58667.85} & \multirow{3}{*}{0} & \multirow{3}{*}{0} & \multirow{3}{*}{1.255} & - & - & . & - & 1499 & \multirow{3}{*}{5999} \\
\hline & & & & & - & - & - & - & 2999 & \\
\hline & & & & & - & - & - & - & 4499 & \\
\hline
\end{tabular}

DWN case study are summarised in Table 1. Simulations have been carried out over a time period of eight days, i.e., $n_{s}=192$ hours, with a sampling time of one hour. The patterns of the applied demands considered in this paper were synthesised from real values reported for this consumption nodes in the Barcelona DWN between July $23^{\text {th }}$ and July $27^{\text {th }}, 2007$. Initial conditions, i.e., source capacities, initial volume of water at tanks and starting demands, are set a priori according to real data. The weights of the cost function (21) are $\lambda_{1}=100, \lambda_{2}=$ 10 , and $\lambda_{3}=1$. The prediction horizon is selected as $N=24$ hours, due to the periodicity of disturbances. The formulation of the optimisation problems and the closedloop simulations have been carried out using MATLAB R2012b (64 bits) and CPLEX solver, running in a PC Intel Core E8600 at $3.33 \mathrm{GHz}$ with $8 \mathrm{~GB}$ of RAM.

The key performance indicators used to assess the aforementioned controllers are defined as follows:

$$
\begin{aligned}
& \Phi_{1} \triangleq \frac{24}{n_{s}} \sum_{k=1}^{n_{s}} \ell_{k}, \\
& \Phi_{2} \triangleq\left|\left\{k \in \mathbb{Z}_{1}^{n_{s}} \mid x_{k}<-B_{p} d_{k}\right\}\right|, \\
& \Phi_{3} \triangleq \sum_{k=1}^{n_{s}} \sum_{i=1}^{n_{x}} \max \left\{0,-B_{p(i)} d_{k}-x_{k(i)}\right\}, \\
& \Phi_{4} \triangleq \frac{1}{n_{s}} \sum_{k=1}^{n_{s}} t_{k},
\end{aligned}
$$

where $\Phi_{1}$ is the average daily multi-objective cost with $\ell_{k}$ given by $(21), \Phi_{2}$ is the number of time instants where water demands are not satisfied (for this, $|\cdot|$ denotes the cardinal of a set of elements), $\Phi_{3}$ is the accumulated volume of water demand that was not satisfied over the simulation horizon, and $\Phi_{4}$ is the average time in seconds required to solve the $\mathrm{MPC}$ problem at each time instant $k \in \mathbb{Z}_{1}^{n_{s}}$. The effect of considering different levels of joint risk acceptability was analysed for the CC-MPC approach, using $\delta=\{0.3,0.2,0.1,0.01,0.001\}$. In the same way, the TB-MPC approach was applied considering different sizes for the initial set of scenarios, i.e., 
$N_{s}=\{19,28,59,599,5999\}$. The size of this initial set was computed following the bound proposed in Schildbach et al. [2013] taking into account the risk levels involved in the chance constraints. This initial set was reduced later by a factor of $0.25,0.50$, and 0.75 to obtain different rooted trees with $N_{r}$ scenarios.

As shown in Table 1, the different CC-MPC scenarios highlight that reliability and control performance are conflicting objectives, i.e., the inclusion of safety mechanisms in the controller increases the reliability of the DWN in terms of demand satisfaction, but also the cost of its operation. The main advantage of the CC-MPC is its formal methodology, which leads to obtain optimal safety constraints that tackle uncertainties and allow to achieve a specified global service level in the DWN. Moreover, the deterministic equivalent CC-MPC robustness is achieved with a low computational burden given that the only extra load (comparing with a nominal formulation) is the computation of the stochastic characteristics of disturbances propagated in the prediction horizon. In this way, the deterministic equivalent CC-MPC approach is suitable for real-time control of large-scale DWNs. Regarding the TB-MPC approach, numeric results show that considering higher $N_{s}$ increments, in average, the stage cost while reducing the volume of unsatisfied water demand. Nevertheless, this latter observation is not applicable for the different $N_{r}$ cases within a same $N_{s}$. This might be influenced by the quality of the information that remains after the scenario generation and reduction algorithms that affect the robustness of the approach and will be subject of further research. The main drawback of the TB-MPC approach is the solution average time and the computational burden. In this case study, the implementation for all cases taking $N_{s}=\{599,5999\}$ was not possible due to memory issues. Hence, some simplification assumptions as those used in Lucia et al. [2013] or parallel computing techniques might be useful.

\section{CONCLUSIONS}

In this paper, two stochastic control approaches have been assessed to deal with the management of a DWN. According to the preliminary results obtained with the considered case study, both techniques show a relatively similar performance. However, it seems clear that CC-MPC is more appropriate when requiring a low probability of constraint violation, because the use of TB-MPC demands the inclusion of a higher number of scenarios, which may be an issue for the application of the latter to largescale DWNs. Future work should include a more detailed study regarding number of scenarios contained in the tree. Likewise, distributed computation could be used in order to relieve the scaling problems of TB-MPC when the number of scenarios is too high. Finally, the assessment of stochastic techniques should be enhanced with the study of other alternatives.

\section{REFERENCES}

G. E. P. Box, G. M. Jenkins, and G. C. Reisnel. Time Series Analysis, Forecasting and Control. Prentice-Hall International, Inc., 1994.

G. Calafiore and F. Dabbene. Probabilistic and randomized methods for design under uncertainty. Springer, 2006.
Giuseppe C. Calafiore, Fabrizio Dabbene, and Roberto Tempo. Research on probabilistic methods for control system design. Automatica, 47(7):1279 - 1293, 2011.

M. Cannon, P. Couchman, and B. Kouvaritakis. MPC for stochastic systems. In R. Findeisen, F. Allgöwer, and L. Biegler, editors, Assessment and Future Directions of Nonlinear Model Predictive Control, volume 358 of Lecture Notes in Control and Information Sciences, pages 255-268. Springer Berlin Heidelberg, 2007.

A. Charnes and W. W. Cooper. Deterministic equivalents for optimizing and satisficing under chance constraints. Operations Research, 11(1):18-39, 1963.

A. Geletu, M. Klöppel, H. Zhang, and P. Li. Advances and applications of chance-constrained approaches to systems optimisation under uncertainty. International Journal of Systems Science, 44(7):1209-1232, 2013.

H. Heitsch and W. Römisch. Scenario tree modeling for multistage stochastic programs. Mathematical Programming, 118(2):371-406, 2009.

S. Lucia, S. Subramanian, and S. Engell. Nonconservative robust nonlinear model predictive control via scenario decomposition. In Proc. 2013 IEEE MultiConference on Systems and Control (MSC), pages 586591, Hyderabad, India, 2013.

J.M. Maciejowski. Predictive control with constraints. Prentice Hall, Essex, England, 2002.

A. Nemirovski and A. Shapiro. Convex approximations of chance constrained programs. SIAM Journal on Optimization, 17(4):969-996, December 2006.

C. Ocampo-Martinez, V. Puig, G. Cembrano, and J. Quevedo. Application of predictive control strategies to the management of complex networks in the urban water cycle. Control Systems, IEEE, 33(1):15-41, 2013.

T.B.M.J. Ouarda and J.W. Labadie. Chance-constrained optimal control for multireservoir system optimization and risk analysis. Stochastic Environmental Research and Risk Assessment, 15:185-204, 2001.

A. Prékopa. Stochastic Programming. Kluwer Academic Publishers, 1995.

L. Raso, P. J. van Overloop, and D. Schwanenberg. Decisions under uncertainty: Use of flexible model predictive control on a drainage canal system. In Proceedings of the 9th Conference on Hydroinformatics, Tianjin, China, 2009.

R.T. Rockafellar and R.J.-B. Wets. Scenario and policy aggregation in optimization under uncertainty. Mathematics of Operation Research, 16(1):119-147, 1991.

G. Schildbach, L. Fagiano, C. Frei, and M. Morari. The scenario approach for stochastic model predictive control with bounds on closed-loop constraint violations. submitted to Automatica, July 2013. URL http: //arxiv.org/abs/1307.5640.

A.T. Schwarm and M. Nikolaou. Chance-constrained model predictive control. AIChE Journal, 45(8):17431752, 1999. 